\title{
Testing the Generalization Power of Neural Network Models Across NLI Benchmarks
}

\author{
Aarne Talman \\ Department of Digital Humanities \\ University of Helsinki \\ Basement AI \\ aarne.talman@helsinki.fi
}

\author{
Stergios Chatzikyriakidis \\ Department of Philosophy, Linguistics and \\ Theory of Science, University of Gothenburg \\ Basement AI \\ stergios.chatzikyriakidis@gu.se
}

\begin{abstract}
Neural network models have been very successful in natural language inference, with the best models reaching $90 \%$ accuracy in some benchmarks. However, the success of these models turns out to be largely benchmark specific. We show that models trained on a natural language inference dataset drawn from one benchmark fail to perform well in others, even if the notion of inference assumed in these benchmarks is the same or similar. We train six high performing neural network models on different datasets and show that each one of these has problems of generalizing when we replace the original test set with a test set taken from another corpus designed for the same task. In light of these results, we argue that most of the current neural network models are not able to generalize well in the task of natural language inference. We find that using large pre-trained language models helps with transfer learning when the datasets are similar enough. Our results also highlight that the current NLI datasets do not cover the different nuances of inference extensively enough.
\end{abstract}

\section{Introduction}

Natural Language Inference (NLI) has attracted considerable interest in the NLP community and, recently, a large number of neural network-based systems have been proposed to deal with the task. One can attempt a rough categorization of these systems into: a) sentence encoding systems, and b) other neural network systems. Both of them have been very successful, with the state of the art on the SNLI and MultiNLI datasets being 90.4\%, which is our baseline with BERT (Devlin et al., 2019), and $86.7 \%$ (Devlin et al., 2019) respectively. However, a big question with respect to these systems is their ability to generalize outside the specific datasets they are trained and tested on. Recently, Glockner et al. (2018) have shown that state-of-the-art NLI systems break considerably easily when, instead of tested on the original SNLI test set, they are tested on a test set which is constructed by taking premises from the training set and creating several hypotheses from them by changing at most one word within the premise. The results show a very significant drop in accuracy for three of the four systems. The system that was more difficult to break and had the least loss in accuracy was the system by Chen et al. (2018) which utilizes external knowledge taken from WordNet (Miller, 1995).

In this paper we show that NLI systems that have been very successful in specific NLI benchmarks, fail to generalize when trained on a specific NLI dataset and then these trained models are tested across test sets taken from different NLI benchmarks. The results we get are in line with Glockner et al. (2018), showing that the generalization capability of the individual NLI systems is very limited, but, what is more, they further show the only system that was less prone to breaking in Glockner et al. (2018), breaks too in the experiments we have conducted.

We train six different state-of-the-art models on three different NLI datasets and test these trained models on an NLI test set taken from another dataset designed for the same NLI task, namely for the task to identify for sentence pairs in the dataset if one sentence entails the other one, if they are in contradiction with each other or if they are neutral with respect to inferential relationship.

One would expect that if a model learns to correctly identify inferential relationships in one dataset, then it would also be able to do so in another dataset designed for the same task. Furthermore, two of the datasets, SNLI (Bowman et al., 2015) and MultiNLI (Williams et al., 2018), have been constructed using the same crowdsourcing approach and annotation instructions (Williams 
et al., 2018), leading to datasets with the same or at least very similar definition of entailment. It is therefore reasonable to expect that transfer learning between these datasets is possible. As SICK (Marelli et al., 2014) dataset has been machineconstructed, a bigger difference in performance is expected.

In this paper we show that, contrary to our expectations, most models fail to generalize across the different datasets. However, our experiments also show that BERT (Devlin et al., 2019) performs much better than the other models in experiments between SNLI and MultiNLI. Nevertheless, even BERT fails when testing on SICK. In addition to the negative results, our experiments further highlight the power of pre-trained language models, like BERT, in NLI.

The negative results of this paper are significant for the NLP research community as well as to NLP practice as we would like our best models to not only to be able to perform well in a specific benchmark dataset, but rather capture the more general phenomenon this dataset is designed for. The main contribution of this paper is that it shows that most of the best performing neural network models for NLI fail in this regard. The second, and equally important, contribution is that our results highlight that the current NLI datasets do not capture the nuances of NLI extensively enough.

\section{Related Work}

The ability of NLI systems to generalize and related skepticism has been raised in a number of recent papers. Glockner et al. (2018) show that the generalization capabilities of state-of-the-art NLI systems, in cases where some kind of external lexical knowledge is needed, drops dramatically when the SNLI test set is replaced by a test set where the premise and the hypothesis are otherwise identical except for at most one word. The results show a very significant drop in accuracy. Kang et al. (2018) recognize the generalization problem that comes with training on datasets like SNLI, which tend to be homogeneous and with little linguistic variation. In this context, they propose to better train NLI models by making use of adversarial examples.

Multiple papers have reported hidden bias and annotation artifacts in the popular NLI datasets SNLI and MultiNLI allowing classification based on the hypothesis sentences alone (Tsuchiya,
2018; Gururangan et al., 2018; Poliak et al., 2018).

Wang et al. (2018) evaluate the robustness of NLI models using datasets where label preserving swapping operations have been applied, reporting significant performance drops compared to the results with the original dataset. In these experiments, like in the BreakingNLI experiment, the systems that seem to be performing the better, i.e. less prone to breaking, are the ones where some kind of external knowledge is used by the model (KIM by Chen et al., 2018 is one of those systems).

On a theoretical and methodological level, there is discussion on the nature of various NLI datasets, as well as the definition of what counts as NLI and what does not. For example, Chatzikyriakidis et al. (2017); Bernardy and Chatzikyriakidis (2019) present an overview of the most standard datasets for NLI and show that the definitions of inference in each of them are actually quite different, capturing only fragments of what seems to be a more general phenomenon.

Bowman et al. (2015) show that a simple LSTM model trained on the SNLI data fails when tested on SICK. However, their experiment is limited to this single architecture and dataset pair. Williams et al. (2018) show that different models that perform well on SNLI have lower accuracy on MultiNLI. However in their experiments they did not systematically test transfer learning between the two datasets, but instead used separate systems where the training and test data were drawn from the same corpora. ${ }^{1}$

\section{Experimental Setup}

In this section we describe the datasets and model architectures included in the experiments.

\subsection{Data}

We chose three different datasets for the experiments: SNLI, MultiNLI and SICK. All of them have been designed for NLI involving three-way classification with the labels entailment, neutral and contradiction. We did not include any datasets with two-way classification, e.g. SciTail (Khot et al., 2018). As SICK is a relatively small dataset with approximately only $10 \mathrm{k}$ sentence pairs, we did not use it as training data in any experiment.

\footnotetext{
${ }^{1}$ To be more precise, Williams et al. (2018) tested some transfer across datasets, but only between MultiNLI and SNLI.
} 


\begin{tabular}{ll|cc}
\hline Train data & Test data & Size of the training set & Size of the test set \\
\hline SNLI & SNLI & 550,152 & 10,000 \\
SNLI & MultiNLI & 550,152 & 20,000 \\
SNLI & SICK & 550,152 & 9,840 \\
MultiNLI & MultiNLI & 392,702 & 20,000 \\
MultiNLI & SNLI & 392,702 & 10,000 \\
MultiNLI & SICK & 392,702 & 9,840 \\
SNLI + MultiNLI & SNLI & 942,854 & 10,000 \\
SNLI + MultiNLI & SICK & 942,854 & 9,840 \\
\hline
\end{tabular}

Table 1: Dataset combinations used in the experiments. The rows in bold are baseline experiments, where the test data comes from the same benchmark as the training and development data.

We also trained the models with a combined SNLI + MultiNLI training set.

For all the datasets we report the baseline performance where the training and test data are drawn from the same corpus. We then take these trained models and test them on a test set taken from another NLI corpus. For the case where the models are trained with SNLI + MultiNLI we report the baseline using the SNLI test data. ${ }^{2}$ All the experimental combinations are listed in Table 1. Examples from the selected datasets are provided in Table 2. To be more precise, we vary three things: training dataset, model and testing dataset. We should qualify this though, since the three datasets we look at, can also be grouped by text domain/genre and type of data collection, with MultiNLI and SNLI using the same data collection style, and SNLI and SICK using roughly the same domain/genre. Hopefully, our set up will let us determine which of these factors matters the most.

We describe the source datasets in more detail below.

\section{SNLI}

The Stanford Natural Language Inference (SNLI) corpus (Bowman et al., 2015) is a dataset of 570k human-written sentence pairs manually labeled with the labels entailment, contradiction, and neutral. The source for the premise sentences in SNLI were image captions taken from the Flickr30k corpus (Young et al., 2014).

\section{MultiNLI}

The Multi-Genre Natural Language Inference (MultiNLI) corpus (Williams et al., 2018) consisting of 433k human-written sentence pairs labeled with entailment, contradiction and neutral.

\footnotetext{
${ }^{2}$ Here we could as well have selected MultiNLI test data However the selection does not impact our findings.
}

MultiNLI contains sentence pairs from ten distinct genres of both written and spoken English. Only five genres are included in the training set. The development and test sets have been divided into matched and mismatched, where the former includes only sentences from the same genres as the training data, and the latter includes sentences from the remaining genres not present in the training data.

We used the matched development set (MultiNLI-m) for the experiments. ${ }^{3}$ The MultiNLI dataset was annotated using very similar instructions as for the SNLI dataset. ${ }^{4}$ Therefore we can assume that the definitions of entailment, contradiction and neutral is the same in these two datasets.

\section{SICK}

SICK (Marelli et al., 2014) is a dataset that was originally constructed to test compositional distributional semantics (DS) models. The dataset contains 9,840 examples pertaining to logical inference (negation, conjunction, disjunction, apposition, relative clauses, etc.). The dataset was automatically constructed taking pairs of sentences from a random subset of the $8 \mathrm{~K}$ ImageFlickr data set (Young et al., 2014) and the SemEval 2012 STS MSRVideo Description dataset (Agirre et al., 2012).

\subsection{Model and Training Details}

We perform experiments with six high-performing models covering the sentence encoding models,

\footnotetext{
${ }^{3}$ Here the choice between MultiNLI matched and mismatched does not make a difference to our experimental setup.

${ }^{4}$ The reason we are not saying "exactly the same" is because in some cases, as the authors report, "the prompts that surround each premise sentence during hypothesis collection are slightly tailored to fit the genre of that premise sentence".
} 


\begin{tabular}{|c|c|}
\hline & entailment \\
\hline SICK & $\begin{array}{l}\text { A person, who is riding a bike, is wearing gear which is black } \\
\text { A biker is wearing gear which is black }\end{array}$ \\
\hline SNLI & $\begin{array}{l}\text { A young family enjoys feeling ocean waves lap at their feet. } \\
\text { A family is at the beach. }\end{array}$ \\
\hline MultiNLI & $\begin{array}{l}\text { Kal tangled both of Adrin's arms, keeping the blades far away. } \\
\text { Adrin's arms were tangled, keeping his blades away from Kal. }\end{array}$ \\
\hline & contradiction \\
\hline SICK & $\begin{array}{l}\text { There is no man wearing a black helmet and pushing a bicycle } \\
\text { One man is wearing a black helmet and pushing a bicycle }\end{array}$ \\
\hline SNLII & $\begin{array}{l}\text { A man with a tattoo on his arm staring to the side with vehicles and buildings behind him. } \\
\text { A man with no tattoos is getting a massage. }\end{array}$ \\
\hline MultiNLI & $\begin{array}{l}\text { Also in Eustace Street is an information office and a cultural center for children, The Ark. } \\
\text { The Ark, a cultural center for kids, is located in Joyce Street. }\end{array}$ \\
\hline & neutral \\
\hline SICK & $\begin{array}{l}\text { A little girl in a green coat and a boy holding a red sled are walking in the snow } \\
\text { A child is wearing a coat and is carrying a red sled near a child in a green and black coat }\end{array}$ \\
\hline SNLI & $\begin{array}{l}\text { An old man with a package poses in front of an advertisement. } \\
\text { A man poses in front of an ad for beer. }\end{array}$ \\
\hline MultiNLI & $\begin{array}{l}\text { Enthusiasm for Disney's Broadway production of The Lion King dwindles. } \\
\text { The broadway production of The Lion King was amazing, but audiences are getting bored. }\end{array}$ \\
\hline
\end{tabular}

Table 2: Example sentence pairs from the three datasets.

cross-sentence attention models as well as finetuned pre-trained language models.

For sentence encoding models, we chose a simple one-layer bidirectional LSTM with max pooling (BiLSTM-max) with the hidden size of 600D per direction, used e.g. in InferSent (Conneau et al., 2017), and HBMP (Talman et al., 2018). For the other models, we have chosen ESIM (Chen et al., 2017), which includes cross-sentence attention, and KIM (Chen et al., 2018), which has cross-sentence attention and utilizes external knowledge. We also selected two model involving a pre-trained language model, namely ESIM + ELMo (Peters et al., 2018) and BERT (Devlin et al., 2019). KIM is particularly interesting in this context as it performed significantly better than other models in the Breaking NLI experiment conducted by Glockner et al. (2018). The success of pre-trained language models in multiple NLP tasks make ESIM + ELMo and BERT interesting additions to this experiment. Table 3 lists the different models used in the experiments.

For BiLSTM-max we used the Adam optimizer (Kingma and $\mathrm{Ba}, 2015$ ), a learning rate of $5 \mathrm{e}-4$ and batch size of 64 . The learning rate was decreased by the factor of 0.2 after each epoch if the model did not improve. Dropout of 0.1 was used between the layers of the multi-layer perceptron classifier, except before the last layer.The BiLSTM-max models were initialized with pre-trained GloVe 840B word embeddings of size 300 dimensions (Pennington et al., 2014), which were fine-tuned during training. Our BiLSMT-max model was implemented in PyTorch.

For HBMP, ESIM, KIM and BERT we used the original implementations with the default settings and hyperparameter values as described in Talman et al. (2018), Chen et al. (2017), Chen et al. (2018) and Devlin et al. (2019) respectively. For BERT we used the uncased 768-dimensional model (BERT-base). For ESIM + ELMo we used the AllenNLP (Gardner et al., 2018) PyTorch implementation with the default settings and hyperparameter values.

\section{Experimental Results}

Table 4 contains all the experimental results.

Our experiments show that, while all of the six models perform well when the test set is drawn from the same corpus as the training and development set, accuracy is significantly lower when we test these trained models on a test set drawn from a separate NLI corpus, the average difference in accuracy being 24.9 points across all experiments.

Accuracy drops the most when a model is tested on SICK. The difference in this case is between 19.0-29.0 points when trained on MultiNLI, between 31.6-33.7 points when trained on SNLI and between 31.1-33.0 when trained on SNLI + MultiNLI. This was expected, as the method of constructing the sentence pairs was different, and hence there is too much difference in the kind of sentence pairs included in the training and test sets for transfer learning to work. However, the drop 


\begin{tabular}{ll}
\hline Model & Model type \\
\hline BiLSTM-max (Conneau et al., 2017) & Sentence encoding \\
HBMP (Talman et al., 2018) & Sentence encoding \\
ESIM (Chen et al., 2017) & Cross-sentence attention \\
KIM (Chen et al., 2018) & Cross-sentence attention \\
ESIM + ELMMo (Peters et al., 2018) & Pre-trained language model \\
BERT-base (Devlin et al., 2019) & Cross-sentence attention + pre-trained language model \\
\hline
\end{tabular}

Table 3: Model architectures used in the experiments.

was more dramatic than expected.

The most surprising result was that the accuracy of all models drops significantly even when the models were trained on MultiNLI and tested on SNLI (3.6-11.1 points). This is surprising as both of these datasets have been constructed with a similar data collection method using the same definition of entailment, contradiction and neutral. The sentences included in SNLI are also much simpler compared to those in MultiNLI, as they are taken from the Flickr image captions. This might also explain why the difference in accuracy for all of the six models is lowest when the models are trained on MultiNLI and tested on SNLI. It is also very surprising that the model with the biggest difference in accuracy was ESIM + ELMo which includes a pre-trained ELMo language model. BERT performed significantly better than the other models in this experiment having an accuracy of $80.4 \%$ and only 3.6 point difference in accuracy.

The poor performance of most of the models with the MultiNLI-SNLI dataset pair is also very surprising given that neural network models do not seem to suffer a lot from introduction of new genres to the test set which were not included in the training set, as can be seen from the small difference in test accuracies for the matched and mismatched test sets (see e.g Williams et al., 2018). In a sense SNLI could be seen as a separate genre not included in MultiNLI. This raises the question if the SNLI and MultiNLI have e.g. different kinds of annotation artifacts, which makes transfer learning between these datasets more difficult.

All the models, except BERT, perform almost equally poorly across all the experiments. Both BiLSTM-max and HBMP have an average drop in accuracy of 24.4 points, while the average for KIM is 25.5 and for ESIM + ELMo 25.6. ESIM has the highest average difference of 27.0 points. In contrast to the findings of Glockner et al. (2018), utilizing external knowledge did not improve the model's generalization capability, as KIM performed equally poorly across all dataset combinations.

Also including a pretrained ELMo language model did not improve the results significantly. The overall performance of BERT was significantly better than the other models, having the lowest average difference in accuracy of 22.5 points. Our baselines for SNLI (90.4\%) and SNLI + MultiNLI (90.6\%) outperform the previous state-of-the-art accuracy for SNLI $(90.1 \%)$ by Kim et al. (2018).

To understand better the types of errors made by neural network models in NLI we looked at some example failure-pairs for selected models. ${ }^{5}$ Tables 5 and 6 contain some randomly selected failurepairs for two models: BERT and HBMP, and for three set-ups: SNLI $\rightarrow$ SICK, SNLI $\rightarrow$ MultiNLI and MultiNLI $\rightarrow$ SICK. We chose BERT as the current the state of the art NLI model. HBMP was selected as a high performing model in the sentence encoding model type. Although the listed sentence pairs represent just a small sample of the errors made by these models, they do include some interesting examples. First, it seems that SICK has a more narrow notion of contradiction - corresponding more to logical contradiction - compared to the contradiction in SNLI and MultiNLI, where especially in SNLI the sentences are contradictory if they describe a different state of affairs. This is evident in the sentence pair: A young child is running outside over the fallen leaves and A young child is lying down on a gravel road that is covered with dead leaves, which is predicted by BERT to be contradiction although the gold label is neutral. Another interesting example is the sentence pair: A boat pear with people boarding and

\footnotetext{
${ }^{5}$ More thorough error analysis of each of the models and set-up is out of scope of this work but we intend to address these in our future research.
} 


\begin{tabular}{|c|c|c|c|c|}
\hline Train data & Test data & Test accuracy & $\Delta$ & Model \\
\hline SNLI & SNLI & 86.1 & & BiLSTM-max (our baseline) \\
\hline SNLI & SNLI & 86.6 & & HBMP (Talman et al., 2018) \\
\hline SNLI & SNLI & 88.0 & & ESIM (Chen et al., 2017) \\
\hline SNLI & SNLI & 88.6 & & KIM (Chen et al., 2018) \\
\hline SNLI & SNLI & 88.6 & & ESIM + ELMo (Peters et al., 2018) \\
\hline SNLI & SNLI & $\underline{90.4}$ & & BERT-base (Devlin et al., 2019) \\
\hline SNLI & MultiNLI-m & $55.7^{*}$ & -30.4 & BiLSTM-max \\
\hline SNLI & MultiNLI-m & $56.3^{*}$ & -30.3 & HBMP \\
\hline SNLI & MultiNLI-m & $59.2^{*}$ & -28.8 & ESIM \\
\hline SNLI & MultiNLI-m & $61.7^{*}$ & -26.9 & KIM \\
\hline SNLI & MultiNLI-m & $64.2^{*}$ & -24.4 & ESIM + ELMo \\
\hline SNLI & MultiNLI-m & $75.5^{*}$ & -14.9 & BERT-base \\
\hline SNLI & SICK & 54.5 & -31.6 & BiLSTM-max \\
\hline SNLI & SICK & 53.1 & -33.5 & HBMP \\
\hline SNLI & SICK & 54.3 & -33.7 & ESIM \\
\hline SNLI & SICK & 55.8 & -32.8 & KIM \\
\hline SNLI & SICK & 56.7 & -31.9 & ESIM + ELMo \\
\hline SNLI & SICK & $\underline{56.9}$ & -33.5 & BERT-base \\
\hline MultiNLI & MultiNLI-m & 73.1 $^{*}$ & & BiLSTM-max \\
\hline MultiNLI & MultiNLI-m & 73.2* & & HBMP \\
\hline MultiNLI & MultiNLI-m & $76.8^{*}$ & & ESIM \\
\hline MultiNLI & MultiNLI-m & $77.3^{*}$ & & KIM \\
\hline MultiNLI & MultiNLI-m & $80.2^{*}$ & & ESIM + ELMo \\
\hline MultiNLI & MultiNLI-m & 84.0 ${ }^{*}$ & & BERT-base \\
\hline MultiNLI & SNLI & 63.8 & -9.3 & BiLSTM-max \\
\hline MultiNLI & SNLI & 65.3 & -7.9 & HBMP \\
\hline MultiNLI & SNLI & 66.4 & -10.4 & ESIM \\
\hline MultiNLI & SNLI & 68.5 & -8.8 & KIM \\
\hline MultiNLI & SNLI & 69.1 & -11.1 & ESIM + ELMo \\
\hline MultiNLI & SNLI & $\underline{80.4}$ & $\underline{-3.6}$ & BERT-base \\
\hline MultiNLI & SICK & 54.1 & -19.0 & BiLSTM-max \\
\hline MultiNLI & SICK & 54.1 & $\overline{-19.1}$ & HBMP \\
\hline MultiNLI & SICK & 47.9 & -28.9 & ESIM \\
\hline MultiNLI & SICK & 50.9 & -26.4 & KIM \\
\hline MultiNLI & SICK & 51.4 & -28.8 & ESIM + ELMo \\
\hline MultiNLI & SICK & $\underline{55.0}$ & -29.0 & BERT-base \\
\hline SNLI + MultiNLI & SNLI & $\overline{86.1}$ & & BiLSTM-max \\
\hline SNLI + MultiNLI & SNLI & 86.1 & & HBMP \\
\hline SNLI + MultiNLI & SNLI & 87.5 & & ESIM \\
\hline SNLI + MultiNLI & SNLI & 86.2 & & KIM \\
\hline SNLI + MultiNLI & SNLI & 88.8 & & ESIM + ELMo \\
\hline SNLI + MultiNLI & SNLI & 90.6 & & BERT-base \\
\hline SNLI + MultiNLI & SICK & 54.5 & -31.6 & BiLSTM-max \\
\hline SNLI + MultiNLI & SICK & 55.0 & $\underline{-31.1}$ & HBMP \\
\hline SNLI + MultiNLI & SICK & 54.5 & $\overline{-33.0}$ & ESIM \\
\hline SNLI + MultiNLI & SICK & 54.6 & -31.6 & KIM \\
\hline SNLI + MultiNLI & SICK & 57.1 & -31.7 & ESIM + ELMo \\
\hline SNLI + MultiNLI & SICK & $\underline{59.1}$ & -31.5 & BERT-base \\
\hline
\end{tabular}

Table 4: Test accuracies (\%). For the baseline results (highlighted in bold) the training data and test data have been drawn from the same benchmark corpus. $\Delta$ is the difference between the test accuracy and the baseline accuracy for the same training set. Results marked with ${ }^{*}$ are for the development set, as no annotated test set is openly available. Best scores with respect to accuracy and difference in accuracy are underlined.

disembarking some boats. and people are boarding and disembarking some boats, which is incorrectly predicted by BERT to be contradiction although it has been labeled as entailment. Here the two sentences describe the same event from different points of view: the first one describing a boat pear with some people on it and the second one describing the people directly. Interestingly the added information about the boat pear seems to confuse the model.

\section{Discussion and Conclusion}

In this paper we have shown that neural network models for NLI fail to generalize across different NLI benchmarks. We experimented with six state-of-the-art models covering sentence en- 
coding approaches, cross-sentence attention models and pre-trained and fine-tuned language models. For all the systems, the accuracy drops between 3.6-33.7 points (the average drop being 24.9 points), when testing with a test set drawn from a separate corpus from that of the training data, as compared to when the test and training data are splits from the same corpus. Our findings, together with the previous negative findings, indicate that the state-of-the-art models fail to capture the semantics of NLI in a way that will enable them to generalize across different NLI situations.

The results highlight two issues to be taken into consideration: a) using datasets involving a fraction of what NLI is, will fail when tested in datasets that are testing for a slightly different definition of inference. This is evident when we move from the SNLI to the SICK dataset. b) NLI is to some extent genre/context dependent. Training on SNLI and testing on MultiNLI gives worse results than vice versa. This is particularly evident in the case of BERT. These results highlight that training on multiple genres helps. However, this help is still not enough given that, even in the case of training on MultiNLI (multi genre) and training on SNLI (single genre and same definition of inference with MultiNLI), accuracy drops significantly.

We also found that involving a large pre-trained language model helps with transfer learning when the datasets are similar enough, as is the case with SNLI and MultiNLI. Our results further corroborate the power of pre-trained and fine-tuned language models like BERT in NLI. However, not even BERT is able to generalize from SNLI and MultiNLI to SICK, possibly due to the difference between what kind of inference relations are contained in these datasets.

Our findings motivate us to look for novel neural network architectures and approaches that better capture the semantics on natural language inference beyond individual datasets. However, there seems to be a need to start with better constructed datasets, i.e. datasets that will not only capture fractions of what NLI is in reality. Better NLI systems need to be able to be more versatile on the types of inference they can recognize. Otherwise, we would be stuck with systems that can cover only some aspects of NLI. On a theoretical level, and in connection to the previous point, we need a better understanding of the range of phenomena NLI must be able to cover and focus our future endeavours for dataset construction towards this direction. In order to do this a more systematic study is needed on the different kinds of entailment relations NLI datasets need to include. Our future work will include a more systematic and broad-coverage analysis of the types of errors the models make and in what kinds of sentence-pairs they make successful predictions.

\section{Acknowledgments}

The first author is supported by the FoTran project, funded by the European Research Council (ERC) erc under the European Unions Horizon 2020 research and innovation programme (grant agreement No 771113).

The first author also gratefully acknowledges the support of the Academy of Finland through project 314062 from the ICT 2023 call on Computation, Machine Learning and Artificial Intelligence.

The second author is supported by grant 201439 from the Swedish Research Council, which funds the Centre for Linguistic Theory and Studies in Probability (CLASP) in the Department of Philosophy, Linguistics, and Theory of Science at the University of Gothenburg.

\section{References}

Eneko Agirre, Mona Diab, Daniel Cer, and Aitor Gonzalez-Agirre. 2012. Semeval-2012 task 6: A pilot on semantic textual similarity. In Proceedings of the First Joint Conference on Lexical and Computational Semantics.

Jean-Philippe Bernardy and Stergios Chatzikyriakidis. 2019. What kind of natural language inference are nlp systems learning: Is this enough? In Proceedings of ICAART.

Samuel R. Bowman, Gabor Angeli, Christopher Potts, and Christopher D. Manning. 2015. A large annotated corpus for learning natural language inference. In Proceedings of EMNLP.

Stergios Chatzikyriakidis, Robin Cooper, Simon Dobnik, and Staffan Larsson. 2017. An overview of natural language inference data collection: The way forward? In Proceedings of the Computing Natural Language Inference Workshop.

Qian Chen, Xiaodan Zhu, Zhen-Hua Ling, Diana Inkpen, and Si Wei. 2018. Neural natural language inference models enhanced with external knowledge. In Proceedings of ACL. 
Qian Chen, Xiaodan Zhu, Zhen-Hua Ling, Si Wei, Hui Jiang, and Diana Inkpen. 2017. Enhanced 1stm for natural language inference. In $A C L$.

Alexis Conneau, Douwe Kiela, Holger Schwenk, Loïc Barrault, and Antoine Bordes. 2017. Supervised learning of universal sentence representations from natural language inference data. In Proceedings of EMNLP.

Jacob Devlin, Ming-Wei Chang, Kenton Lee, and Kristina Toutanova. 2019. Bert: Pre-training of deep bidirectional transformers for language understanding. In Proceedings of NAACL.

Matt Gardner, Joel Grus, Mark Neumann, Oyvind Tafjord, Pradeep Dasigi, Nelson F. Liu, Matthew Peters, Michael Schmitz, and Luke S. Zettlemoyer. 2018. AllenNLP: A deep semantic natural language processing platform. In ACL workshop for NLP Open Source Software.

Max Glockner, Vered Shwartz, and Yoav Goldberg. 2018. Breaking nli systems with sentences that require simple lexical inferences. In Proceedings of $A C L$.

Suchin Gururangan, Swabha Swayamdipta, Omer Levy, Roy Schwartz, Samuel Bowman, and Noah A. Smith. 2018. Annotation artifacts in natural language inference data. In Proceedings of NAACL.

Dongyeop Kang, Tushar Khot, Ashish Sabharwal, and Eduard Hovy. 2018. Adversarial training for textual entailment with knowledge-guided examples. In Proceedings of ACL.

Tushar Khot, Ashish Sabharwal, and Peter Clark. 2018. Scitail: A textual entailment dataset from science question answering. In Proceedings of AAAI.

Seonhoon Kim, Jin-Hyuk Hong, Inho Kang, and Nojun Kwak. 2018. Semantic sentence matching with densely-connected recurrent and co-attentive information. arXiv preprint arXiv:1805.11360.

Diederik P. Kingma and Jimmy Ba. 2015. Adam: A method for stochastic optimization. In Proceedings of ICLR.

Marco Marelli, Stefano Menini, Marco Baroni, Luisa Bentivogli, Raffaella Bernardi, and Roberto Zamparelli. 2014. A sick cure for the evaluation of compositional distributional semantic models. In Proceedings of LREC.

George A. Miller. 1995. Wordnet: A lexical database for english. Commun. ACM, 38(11):39-41.

Jeffrey Pennington, Richard Socher, and Christopher D. Manning. 2014. Glove: Global vectors for word representation. In Proceedings of EMNLP.

Matthew E. Peters, Mark Neumann, Mohit Iyyer, Matt Gardner, Christopher Clark, Kenton Lee, and Luke Zettlemoyer. 2018. Deep contextualized word representations. In Proceedings of NAACL.
Adam Poliak, Jason Naradowsky, Aparajita Haldar, Rachel Rudinger, and Benjamin Van Durme. 2018. Hypothesis only baselines in natural language inference. In Proceedings of the Seventh Joint Conference on Lexical and Computational Semantics.

Aarne Talman, Anssi Yli-Jyrä, and Jörg Tiedemann. 2018. Natural language inference with hierarchical bilstm max pooling architecture. arXiv preprint arXiv:1808.08762.

Masatoshi Tsuchiya. 2018. Performance Impact Caused by Hidden Bias of Training Data for Recognizing Textual Entailment. In Proceedings of LREC.

Haohan Wang, Da Sun, and Eric P. Xing. 2018. What if we simply swap the two text fragments? a straightforward yet effective way to test the robustness of methods to confounding signals in nature language inference tasks. arXiv preprint arXiv:1809.02719.

Adina Williams, Nikita Nangia, and Samuel R. Bowman. 2018. A broad-coverage challenge corpus for sentence understanding through inference. In Proceedings of NAACL.

Peter Young, Alice Lai, Micah Hodosh, and Julia Hockenmaier. 2014. From image descriptions to visual denotations: New similarity metrics for semantic inference over event descriptions. TACL, 2:6778 
BERT: SNLI $\rightarrow$ SICK

A young child is running outside over the fallen leaves

A young child is lying down on a gravel road that is covered with dead leaves

Label: neutral Prediction: contradiction

The man is being knocked off of a horse

Someone is falling off a horse

Label: entailment Prediction: contradiction

There is no one typing

Someone is typing on a keyboard

Label: contradiction Prediction: neutral

The man in the purple hat is operating a camera that makes videos

There is no man with a camera studying the subject

Label: neutral Prediction: contradiction

A woman is taking off a cloak, which is very large, and revealing an extravagant dress

A woman is putting on a cloak, which is very large, and concealing an extravagant dress

Label: contradiction Prediction: neutral

BERT: MultiNLI $\rightarrow$ SICK

A cowboy is riding a horse and cornering a barrel

A cowgirl is riding a horse and corners a barrel

Label: neutral Prediction: contradiction

A tan dog is jumping up and catching a tennis ball

A dog with a tan coat is jumping up and catching a tennis ball

Label: entailment Prediction: neutral

The bunch of men are playing rugby on a muddy field

Some men are idling

Label: neutral Prediction: contradiction

A blond child is going down a slide and throwing up his arms

A child with dark hair is going down a slide and throwing up his arms

Label: contradiction Prediction: entailment

There is no person in bike gear standing steadily in front of the mountains

A group of people is equipped with protective gear

Label: neutral Prediction: contradiction

BERT: MultiNLI $\rightarrow$ SNLI

A woman in a white wedding dress is being dressed and fitted by two other women.

A woman is being fitted for the first time.

Label: neutral Prediction: contradiction

A boat pear with people boarding and disembarking some boats.

people are boarding and disembarking some boats

Label: entailment Prediction: contradiction

Several men at a bar watch a sports game on the television.

The men are at a baseball game.

Label: contradiction Prediction: entailment

A singer wearing a leather jacket performs on stage with dramatic lighting behind him.

a singer is on american idol

Label: neutral Prediction: contradiction

A person rolls down a hill riding a wagon as another watches.

A person stares at an empty hill.

Label: contradiction Prediction: neutral

Table 5: Example failure-pairs for BERT. 
HBMP: SNLI $\rightarrow$ SICK

a boy is sitting in a room and playing a piano by lamp light

a boy is playing a keyboard

Label: entailment Prediction: contradiction

a woman is wearing ear protection and is firing a gun at an outdoor shooting range

a woman is shooting at target practices

Label: entailment Prediction: neutral

a man in a purple hat isn't climbing a rocky wall with bare hands

a man in a purple hat is climbing a rocky wall with bare hands

Label: contradiction Prediction: entailment

a cat is swinging on a fan

a cat is stuck on a moving ceiling fan

Label: neutral Prediction: contradiction

a young boy is jumping and covering nearby wooden fence with grass

a young boy covered in grass is jumping near a wooden fence

Label: neutral Prediction: entailment

HBMP: MultiNLI $\rightarrow$ SICK

two dogs are walking slowly through a park

two dogs are running quickly through a park

Label: neutral Prediction: entailment

the woman is playing a guitar which is electric

the woman is playing an electric guitar

Label: entailment Prediction: neutral

there is no man squatting in brush and taking a photograph

a man is crouching and holding a camera

Label: neutral Prediction: contradiction

the snowboarder is jumping off a snowy hill

a snowboarder is jumping off the snow

Label: neutral Prediction: entailment

the boy is sitting near the blue ocean

the boy is wading through the blue ocean

Label: contradiction Prediction: neutral

HBMP: MultiNLI $\rightarrow$ SNLI

a man is holding a book standing in front of a chalkboard.

a person is in a classroom teaching.

Label: entailment Prediction: contradiction

a woman with a pink purse walks down a crowded sidewalk.

a woman escapes a from a hostile enviroment

Label: neutral Prediction: contradiction

a woman with a pink purse walks down a crowded sidewalk.

a woman escapes a from a hostile enviroment

Label: neutral Prediction: contradiction

a person waterskiing in a river with a large wall in the background.

a dog waterskiing in a river with a large wall in the background.

Label: contradiction Prediction: neutral

a man wearing a blue shirt and headphones around his neck raises his arm.

a man is raising his arm to get someones attention.

Label: neutral

Prediction: entailment

Table 6: Example failure-pairs for HBMP. 